\title{
Significance and Diagnostic Accuracy of Early S100B Serum Concentration after Aneurysmal Subarachnoid Hemorrhage
}

\author{
Baptiste Balança ${ }^{1,2,3, *,+} \mathbb{\infty}$, Thomas Ritzenthaler ${ }^{1,+}$, Florent Gobert ${ }^{1}$, Caroline Richet ${ }^{4}(\mathbb{D}$, \\ Carole Bodonian ${ }^{1}$, Romain Carrillon ${ }^{1}$, Anne Terrier ${ }^{1}$, Laurent Desmurs ${ }^{4}$, \\ Armand Perret-Liaudet ${ }^{4,5}$ and Frédéric Dailler ${ }^{1}$ \\ 1 Hospices Civils de Lyon, Hôpital Pierre Wertheimer, Department of Neurological Anesthesiology and \\ Intensive Care Medicine, 69500 Lyon, France; thomas.ritzenthaler@chu-lyon.fr (T.R.); \\ Florent.GOBERT01@chu-lyon.fr (F.G.); carole.bodonian@chu-lyon.fr (C.B.); \\ romain.carrillon@chu-lyon.fr (R.C.); anne.terrier@chu-lyon.fr (A.T.); frederic.dailler@chu-lyon.fr (F.D.) \\ Inserm U1028, CNRS UMR 5292, Lyon Neuroscience Research Centre, Team TIGER, 69500 Lyon, France \\ 3 Université Claude Bernard Lyon 1, Centre Lyonnais d'Enseignement par la Simulation en Santé, SAMSEI, \\ 69008 Lyon, France \\ 4 Hospices Civils de Lyon, Hôpital Pierre Wertheimer, Clinical Chemistry and Molecular Biology Laboratory, \\ 69500 Lyon, France; caroline.richet@chu-lyon.fr (C.R.); laurent.desmurs@chu-lyon.fr (L.D.); \\ armand.perret-liaudet@chu-lyon.fr (A.P.-L.) \\ 5 Inserm U1028, CNRS UMR 5292, Lyon Neuroscience Research Centre, Team BIORAN, 69500 Lyon, France \\ * Correspondence: baptiste.balanca@chu-lyon.fr; Tel.: +33-4-72-35-75-76 \\ $\dagger$ These authors contributed equally to this work.
}

Received: 13 May 2020; Accepted: 3 June 2020; Published: 5 June 2020

\begin{abstract}
Background: Early brain injuries (EBI) are one of the most important causes of morbidity and mortality after subarachnoid hemorrhage. At admission, a third of patients are unconscious (spontaneously or sedated) and EBI consequences are not evaluable. To date, it is unclear who will still be comatose (with severe EBI) and who will recover (with less severe EBI) once the aneurysm is treated and sedation withdrawn. The objective of the present study was to determine the diagnostic accuracy of S100B levels at hospital admission to identify patients with severe neurological consequences of EBI. Methods: Patients were consecutively included in this prospective blinded observational study. A motor component of the Glasgow coma score under 6 on day 3 was used to define patients with severe neurological consequences of EBI. Results: A total of 81 patients were included: 25 patients were unconscious at admission, 68 were treated by coiling. On day 3, 12 patients had severe consequences of EBI. A maximal S100B value between admission and day 1 had an area under the receiver operating characteristic curve (AUC) of $86.7 \%$ to predict severe EBI consequences. In patients with impaired consciousness at admission, the AUC was $88.2 \%$. Conclusion: Early S100B seems to have a good diagnostic value to predict severe EBI. Before claiming the usefulness of S100B as a surrogate marker of EBI severity to start earlier multimodal monitoring, these results must be confirmed in an independent validation cohort.
\end{abstract}

Keywords: S100B protein; subarachnoid hemorrhage; early brain injury; patient outcome assessment

\section{Introduction}

The 28-day mortality associated with subarachnoid hemorrhage (SAH) is reported to range from $26 \%$ to $40 \%$ in Europe [1] and half of those who survive sustain irreversible brain damage [2]. Bleeding consequences, such as cerebral edema, intracerebral hemorrhage, mechanical stress, or raised 
intracranial pressure, as well as re-bleeding or aneurysm treatment complications, are important determinants of poor outcome [3-7]. These first brain insults have been grouped together and termed early brain injuries (EBI). Physicians usually rate EBI at two critical moments: at the first physician contact using the World Federation of Neurosurgical Surgeons (WFNS) grading scale, which is a good predictor of clinical outcome [8]; and 3 days later, once the aneurysm is secured, potential hydrocephalus is treated, anesthesia is withdrawn, and before the period during which delayed cerebral ischemia (DCI) can occur, as the latter impacts prognosis $[9,10]$. At day 3 , patients without any loss of consciousness or neurological deficit, supposed to have no or mild EBI, can be differentiated from unconscious patients suffering severe EBI $[9,10]$. Since severe EBI increases the risk of DCI, it is important to identify early on those who require multimodal monitoring to detect delayed injury [11-13]. Yet, the consequences of EBI may not be assessable by clinical examination, as many patients require sedation before or at admission to the intensive care unit (ICU; e.g., due to respiratory failure, early seizure or loss of consciousness).

Several early markers have been used to rate EBI after SAH. For instance, the presence of a cerebral edema on computed tomography (CT) images, graded using the subarachnoid hemorrhage early brain edema score (SEBES), or the extent of fluid attenuation inversion recovery (FLAIR) hypersignals on magnetic resonance imaging, are predictors of long-term disability and DCI occurrence [6,7,14]. Systemic biomarkers have also been evaluated to rate the burden of brain injury, such as the neuron specific enolase (NSE) following cardiac arrest $[15,16]$. However, in hemorrhagic stroke, astrocytic markers are better biomarkers of the primary hemorrhagic lesion [17-19]. The most studied is S100B, a calcium-binding protein expressed predominantly in astroglial cells. A high serum S100B level during the first days after SAH is also predicative of a poor long-term outcome, and is increased by cerebral infarction $[18,20,21]$. However, the long-term outcome is the result of both early and delayed injury, and it is unclear whether S100B can be used to rate EBI severity. In the absence of an early biomarker to identify patients who will remain unconscious (i.e., with severe EBI) 3 days after the bleeding, we hypothesized that early S100B serum concentration reflects the extent of EBI caused by the primary hemorrhage.

The primary aim of this study was therefore to evaluate the diagnostic accuracy of S100B to predict the severe consequences of the primary hemorrhage. We chose a pragmatic clinical approach and defined nonconscious patients at day 3 as having severe consequences of EBI. We also explored early S100B diagnostic accuracy to predict the outcome at ICU discharge.

\section{Experimental Section}

This prospective blinded single-center observational study was conducted in the neurological department of anesthesiology and intensive care medicine of the Pierre Wertheimer Hospital (Hospices Civils de Lyon, Lyon, France). S100B measurements were not available to the clinical staff and therefore did not influence therapeutic choice or any outcome assessment. Written and oral information was given to the patients or their relatives. This study is part of the single-center Prospective, Observational Registry of Patient with Subarachnoid Hemorrhage in Neurocritical Care Unit (ProReSHA) which was approved by our local ethics committee (Comité de Protection des Personnes Lyon Sud Est II, nºRB: 00009118), and registered on clinicaltrials.org (NCT02890004).

\subsection{Participants}

The ProReSHA started in September 2016. Since S100B measurements were available in our hospital on 28 November 2016, the patients of the study reported herein were consecutively included from this date to 23 November 2017. Inclusion criteria were admission for a recent clinical history of $\mathrm{SAH}$ with evidence of bleeding on $\mathrm{CT}$ and age $\geq 18$ years. Exclusion criteria were traumatic $\mathrm{SAH}$, the absence of aneurysm on first angiography, a delay $>36 \mathrm{~h}$ between the ictus and ICU admission, and ongoing sedation at day 3. 


\subsection{Outcome}

The definition of EBI can vary depending on the method of evaluation (i.e., clinical evaluation, brain imaging, invasive neuro-monitoring, neuroinflammation, electrophysiology) [10]. We chose a pragmatic approach usable at the bedside and tried to differentiate patients without or with mild EBI (e.g., without loss of consciousness) from those with moderate EBI (i.e., with an initial loss of consciousness that will rapidly recover) and those with high EBI who will remain unconscious for several days. Although the WFNS score is used to assess patient severity at first physician contact, it was not designed to monitor clinical changes during the hospital stay. Instead, the Glasgow coma scale (GCS) is used to monitor the neurological state and look for a new neurological deficit after SAH [22]. Therefore, its motor component (M-GCS) on day 3 was chosen as the reference for early outcome, as M-GCS is both widely used and reproducible at the bedside and because, at day 3, patients are expected to have received aneurysm treatment, with or without hydrocephalus treatment, and anesthesia should have been withdrawn. A score $<6$ on the M-GCS indicates an unconscious patient who does not respond to an oral command. Based on the M-GCS at ICU admission and day 3, we defined three groups: the EBI-mild group for whom the M-GCS was 6 at arrival and day 3, the EBI-moderate group for patients with an M-GCS $<6$ at arrival and 6 on day 3, and the EBI-severe group presenting an M-GCS score $<6$ at day 3 regardless of the M-GCS on arrival.

\subsection{Clinical Evaluation}

The physicians and nurses in charge of patients and the clinical data assessors were blinded to the S100B levels. SAH severity was evaluated using the WFNS at first contact with a physician (emergency room or out of hospital) and the GCS at admission to the ICU [8]. The Fisher grade [23,24], the SEBES [14], and the Hijdra score [25] were also collected from the first CT. On day 3, the best motor, eye, and verbal GCS sub-scores were collected from the daily evaluation. Other clinical and laboratory parameters, as well as medications, were available hourly from the IntelliSpace Critical Care and Anesthesia software (Philips Informatique Médicale, Suresnes, France), as well as the presence of sedation and hemodynamic or ventilation support. The assessor of the modified Rankin Scale (mRS) at ICU discharge was also blinded to the S100B levels.

\subsection{Clinical Management}

Patients were managed per international SAH treatment guidelines [2]. Briefly, they received daily oral nimodipine (60 mg every $4 \mathrm{~h}$ ). Coiling was usually preferred to clipping, except in anatomical conformations that prevented coiling from being used or when intraparenchymal hematoma required surgical evacuation [26]. In the case of hydrocephalus observed on CT, an external ventricular drainage (EVD, Integra Life Science, Saint Priest, France) was inserted. Blood glucose was kept between 8 and $10 \mathrm{mmol} / \mathrm{L}$, systolic arterial pressure between 120 and $160 \mathrm{mmHg}$ before aneurysm repair, $\mathrm{SpO}_{2}$ above $96 \%$, and in the case of mechanical ventilation, carbon dioxide partial pressure was kept between 35 and $40 \mathrm{mmHg}$. An arterial and a central venous catheter were used if patients needed noradrenaline or mechanical ventilation (with or without sedation).

\subsection{S100B Measurements}

Blood samples were collected at admission and on day 1 . They were centrifuged in the biology laboratory and frozen at $-20{ }^{\circ} \mathrm{C}$ for further analyses. S100B serum levels were measured with an electroluminescent immunoassay kit using a sandwich technique (Cobas, Roche, Mannheim, Germany). This allows the measurement of S100B concentrations between 0.005 and $39 \mu \mathrm{g} / \mathrm{L}$; the limit of quantification is $0.02 \mu \mathrm{g} / \mathrm{L}$. The within-assay coefficient of variation $(\mathrm{CV})$ was $2.1 \%$ at $0.059 \mu \mathrm{g} / \mathrm{L}$ and $0.9 \%$ at $0.372 \mu \mathrm{g} / \mathrm{L}$ mean concentrations. The between-assay CV was $6.2 \%$ at $0.200 \mu \mathrm{g} / \mathrm{L}$ and $3.2 \%$ at $2.430 \mu \mathrm{g} / \mathrm{L}$ mean concentrations. 


\subsection{Statistical Analysis}

This study follows the standards for reporting diagnostic accuracy studies (STARD) 2015 recommendations for analyses of biomarker diagnostic accuracy [27]. The necessary number of subjects was calculated using the Obuchowski method with the assumption of a ratio of one case (patients with M-GCS $<6$ at day 3) to seven controls (patients with M-GCS $=6$ at day 3) [28]. A minimum total population of 64 patients, including eight cases (patients with M-GCS $<6$ at day 3 ) was necessary to detect an area under the receiver operating characteristic (ROC) curve (AUC) $>0.8$, with a power of 0.8 and an alpha risk of 0.05 .

Data were expressed as their median and interquartile range (IQR). Comparisons between the groups were carried out using the Kruskal-Wallis test and post hoc analyses using the Bonferroni-Dunn test. Comparisons of proportions were made using Fisher's exact test, with Bonferroni correction for multiple comparisons.

ROC efficiency functions were calculated using the pROC library [29]. The reference standard (M-GCS at day 3) was chosen before the statistical analyses. The best threshold values were calculated according to the point closest to the ROC curve's top-left point (i.e., optimization of: $\min \left(\left(1\right.\right.$ - sensitivity) ${ }^{2}$ $\left.+(1 \text {-specificity })^{2}\right)$. The $95 \%$ confidence intervals $(95 \% \mathrm{CI})$ were calculated using a bootstrapping method. The AUCs were compared using the Delong test. The effect size of the EBI-severe group on S100B levels was evaluated by the Cliff's delta statistic because of a non-normal distribution (approaching 0 meaning a small effect size and 1 meaning a large effect size) [30]. The AUCs of a combination of EBI markers was evaluated with a logistic regression model, including the following predictors: S100B, SEBES, Hijdra, the presence of an intracerebral hematoma, and GCS at ICU arrival. The dependent variable was the presence or absence of severe EBI at day 3 (i.e., M-GCS $<6$ ).

Biomarkers help to stratify the risk of a condition and guide clinical decision making. Therefore, rather than a single cut-off that dichotomizes the population, another approach is to assess a gray zone with diagnostic uncertainty, allowing for a certain continuum in risk stratification. The first cut-off is chosen to include the diagnosis with near-certainty (i.e., privileging sensitivity); the second is chosen to exclude the diagnosis with near-certainty (i.e., privileging specificity). When the values of the biomarker fall into the gray zone between the two cut-offs, uncertainty exists, and the physician should pursue a diagnosis using additional tools. Therefore, the zone of diagnostic uncertainty, or gray zone, was defined from the first values with a sensitivity or specificity of at least $90 \%$ [31].

A value of $p<0.05$ was considered significant. Statistical analyses were performed using the $R$ software (R Foundation for Statistical Computing, Vienna, Austria, version 3.3.1) [32].

\section{Results}

\subsection{Population}

A total of 132 patients were potentially eligible, and 81 were included in the analyses (Figure 1). For $84 \%$ of them, aneurysm was treated by coiling within a median (IQR) of $14 \mathrm{~h} \mathrm{[5-21].} \mathrm{At} \mathrm{ICU}$ admission, 56 patients had an M-GCS of 6, among whom 53 had an M-GCS of 6 both at admission and on day 3; three patients worsened within 3 days (two had hydrocephalus requiring EVD and the third was intubated for respiratory disorder; none had re-bleeding or aneurysm treatment complications). According to the M-GCS at admission and day 3, 53 patients were classified as EBI-mild (M-GCS $=6$ at admission and day 3), 16 as EBI-moderate (M-GCS $<6$ at admission and M-GCS $=6$ at day 3), and 12 as EBI-severe (M-GCS $<6$ at day 3 regardless of the M-GCS on admission). In the EBI-severe group, the median M-GCS at day 3 was 4/6 [4,5] (i.e., M-GCS of 5: $n=4 / 12 ; 4: n=4 / 12 ; 2: n=1 / 12 ; 1: n=3 / 12$ ). At day 3 , no patients were sedated or had seizures, and all had been treated for hydrocephalus.

The three groups were significantly different in terms of the simplified gravity index (SAPSS), anti-coagulant treatment, WFNS, Fisher grade, Hijdra score, and length of stay, as well as the frequency of hydrocephalus, re-bleeding, and sedation need. Re-bleeding was a rare event $(n=4)$ and occurred 
within $24 \mathrm{~h}$ after admission to the ICU. Patients in the EBI-severe and EBI-moderate groups had a worse outcome at ICU discharge compared to the EBI-mild group (Table 1).

Table 1. Patient characteristics according to EBI group.

\begin{tabular}{|c|c|c|c|c|c|}
\hline & $\begin{array}{c}\text { All } \\
(n=81)\end{array}$ & $\begin{array}{l}\text { EBI-Mild } \\
(n=53)\end{array}$ & $\begin{array}{l}\text { EBI-Moderate } \\
\quad(n=16)\end{array}$ & $\begin{array}{l}\text { EBI-Severe } \\
\qquad(n=12)\end{array}$ & $p$-Value \\
\hline \multicolumn{6}{|l|}{ Demographic data } \\
\hline Sex, male, $n(\%)$ & $29(36 \%)$ & $23(43 \%)$ & $4(25 \%)$ & $2(17 \%)$ & 0.141 \\
\hline Median SAPSS, (IQR) & $25(19-39)$ & $22(17-27)^{* * *}$ & $41(28-54) ¥ ¥ ¥$ & $58(39-71)$ & $<0.001$ \\
\hline Median size, mm (IQR) & $4.8(3.5-7.7)$ & $4.7(3.6-7.6)$ & $4.5(3.3-8.8)$ & $4.8(4-6.7)$ & 0.987 \\
\hline \multicolumn{6}{|l|}{ Location, $n(\%)$} \\
\hline $\begin{array}{l}\text { anterior circulation (ACA, ACoA, AChA, } \\
\text { pericalosal) }\end{array}$ & $29(36 \%)$ & $21(40 \%)$ & $2(13 \%)$ & $6(50 \%)$ & 0.094 \\
\hline posterior circulation (PCA, PCoA) & $8(10 \%)$ & $5(9 \%)$ & $2(13 \%)$ & $1(8 \%)$ & 0.851 \\
\hline Hydrocephalus, $n(\%)$ & $30(37 \%)$ & $11(21 \%)$ & $12(75 \%) ¥ ¥ ¥$ & $7(58 \%)$ & $<0.001$ \\
\hline Sedation at admission, $n(\%)$ & $23(28 \%)$ & $2(4 \%)^{* * *}$ & $12(75 \%) ¥ ¥ ¥$ & $9(75 \%)$ & $<0.001$ \\
\hline Re-bleeding, $n(\%)$ & $4(5 \%)$ & $0^{* *}$ & $1(6 \%)$ & $3(25 \%)$ & 0.003 \\
\hline Aneurysm treatment, number of coiling, $n(\%)$ & $68(84 \%)$ & $47(89 \%)$ & $13(81 \%)$ & $8(67 \%)$ & 0.134 \\
\hline $\begin{array}{l}\text { Median time from admission to treatment, } \\
\mathrm{h}(\mathrm{IQR})\end{array}$ & $14(5-21)$ & $14(8-20)$ & $14(3-21)$ & $43(15-68)$ & 0.150 \\
\hline Aneurysm treatment complication, $n(\%)$ & $15(19 \%)$ & $8(15 \%)$ & $5(31 \%)$ & $2(17 \%)$ & 0.259 \\
\hline \multicolumn{6}{|l|}{ WFNS at first physician contact, $n(\%)$} \\
\hline SEBES > 2, $n(\%)$ & $64(79 \%)$ & $39(73.6 \%)$ & $14(87.5 \%)$ & $11(91.7 \%)$ & 0.305 \\
\hline $\mathrm{ICH}, n(\%)$ & $17(21 \%)$ & $8(15.1 \%)$ & $4(25 \%)$ & $5(41.7 \%)$ & 0.107 \\
\hline Median GCS at ICU admission, (IQR) & $14(3-15)$ & $15(14-15)$ & $3(3-4)$ & $3(3-6)$ & $<0.001$ \\
\hline DCI occurrence, $n(\%)$ & $20(25 \%)$ & $10(19 \%)$ & $7(44 \%)$ & $3(25 \%)$ & 0.116 \\
\hline Median mRS at ICU discharge (IQR) & $3(1-4)$ & $2(1-3)^{* * *}$ & $3(3-4) * ¥$ & $5(5-6)$ & $<0.001$ \\
\hline Median length of ICU stay, days (IQR) & $11(9-16)$ & $10(9-13)$ & $16(13-18) ¥ ¥$ & $11(4-18)$ & 0.012 \\
\hline
\end{tabular}

The three groups were defined according to M-GCS at ICU admission and day 3: EBI-mild M-GCS $=6$ at arrival and day 3, EBI-moderate M-GCS $<6$ at arrival and $=6$ on day 3, EBI-severe M-GCS $<6$ both at arrival and day 3. Results of post hoc tests: Differences compared to the EBI-severe group, ${ }^{*} p<0.05^{* *} p<0.01^{* * *} p<0.001$; differences between EBI-moderate and EBI-mild groups, $¥ p<0.05 ¥ p<0.01 ¥ ¥ ¥ p<0.001$. GCS: Glasgow coma scale, OAD: oral antidiabetic drug, ACA: anterior cerebral artery, ACoA: anterior communicative artery, AChA: choroidal artery, PCA: posterior cerebral artery, PCoA: posterior communicative artery, MCA: mean cerebral artery, DCI: delayed cerebral ischemia, ICH: intracerebral hematoma; mRS: modified Rankin Scale. 


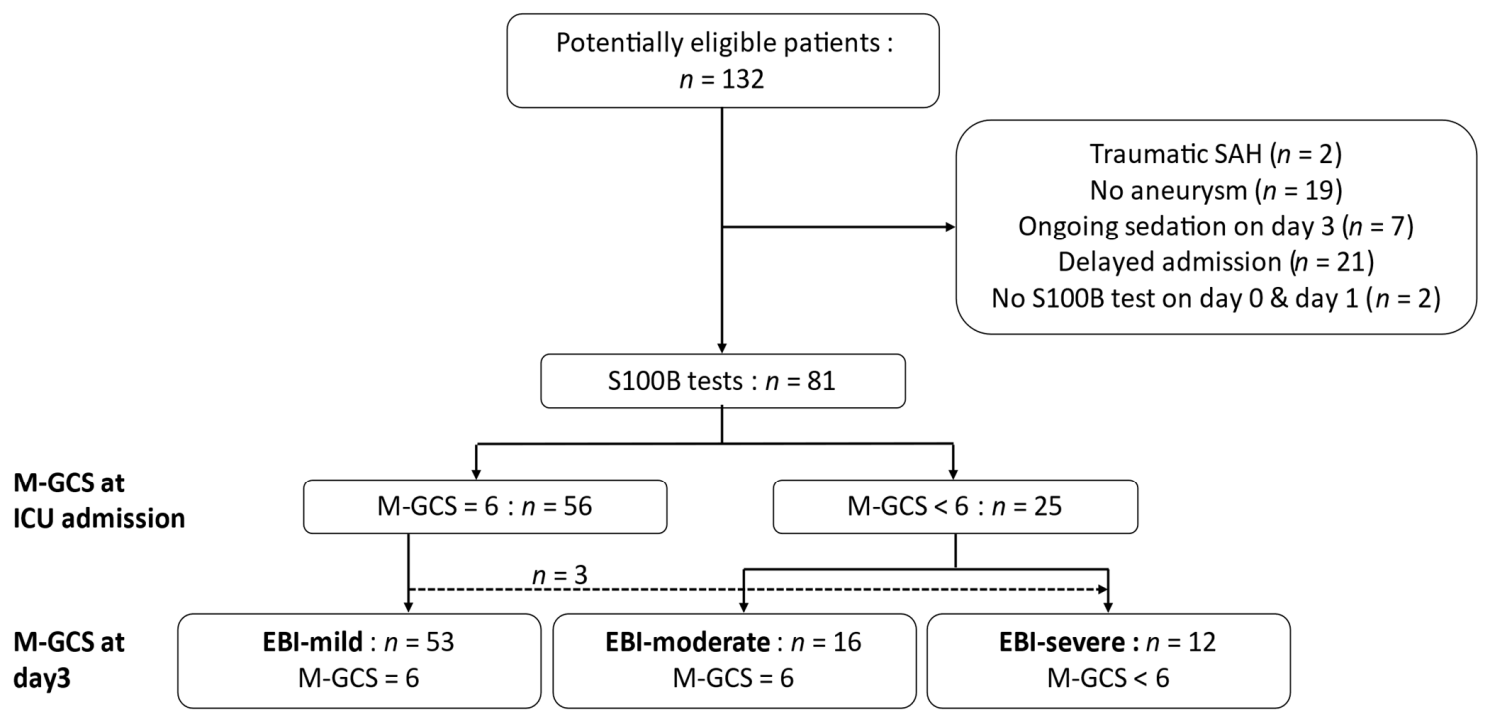

Figure 1. Flow chart. EBI: Early brain injury, GCS: Glasgow coma scale, M-GCS: motor score of GCS, SAH: subarachnoid hemorrhage. Patients' clinical evolution over the first 3 days and their affiliation to the EBI-mild, EBI-moderate, and EBI-severe groups is presented. Among the three patients who worsened within 3 days, two had hydrocephalus requiring external ventricular drainage (EVD). The third was intubated for respiratory disorder, her M-GCS at day 3 was 5 without sedation and her modified Rankin Scale (mRS) at intensive care unit discharge was 3 (i.e., moderate disability). None had re-bleeding or aneurysm treatment complications.

\subsection{Serum S100B Concentration Changes}

We were able to assess the S100B time course in 61 patients with both measurements at admission and on day 1 (EBI-severe: $n=11$, EBI-moderate: $n=13$, EBI-mild: $n=37$ ). There were no significant differences between admission and day 1 (median (IQR), respectively, $0.098 \mu \mathrm{g} / \mathrm{L}(0.067-0.185) \mathrm{vs}$. $0.100 \mu \mathrm{g} / \mathrm{L}(0.069-0.172), p=0.501)$. Moreover, 46 patients had daily measurements from admission to day 3. The median (IQR) S100B value declined significantly on days $2(p=0.003)$ and $3(p<0.001)$ compared to admission (admission: $0.094 \mu \mathrm{g} / \mathrm{L}(0.061-0.176)$; day 1: $0.092 \mu \mathrm{g} / \mathrm{L}(0.068-0.169)$; day 2: $0.085 \mu \mathrm{g} / \mathrm{L}(0.057-0.112)$; day 3: $0.064 \mu \mathrm{g} / \mathrm{L}(0.048-0.09)$.

\subsection{Accuracy of Serum S100B Concentration to Predict an M-GCS $<6$ on Day 3}

We included 81 patients with a $\geq 1$ S100B measurement at admission or day 1 and used their maximal value to evaluate the S100B diagnostic accuracy to predict a M-GCS $<6$ at day 3 . The median S100B value was higher in the EBI-severe group $(0.467 \mu \mathrm{g} / \mathrm{L}$, IQR $(0.171-1.09))$ than in the EBI-moderate $(0.134 \mu \mathrm{g} / \mathrm{L}$, IQR $(0.092-0.204) ; p=0.022)$ or the EBI-mild group $(0.098 \mu \mathrm{g} / \mathrm{L}$, IQR $(0.068-0.138) ; p<0.001)$. There was a large effect size of the EBI-severe group vs. the others on the maximal S100B value at admission or day 1 , as evidenced by a Cliff's delta of $0.73(95 \%$ CI $(0.46 ; 0.88))$. The S100B AUC was not significantly different from that of the GCS at ICU admission $(86.7 \%(95 \%$ CI $(73.6 ; 95.9)$ vs. $84.1 \%$, 95\% CI (73.9-92.6), $p=0.573)$, and tended to be higher than the AUC of the Hijdra score $(68.03 \%$ $95 \%$ CI $(51.4 ; 82.2), p=0.057)$, as well as the SEBES $(68 \% 95 \%$ CI $(52.1 ; 81.7), p=0.054)$. The AUC of a logistic regression model, including S100B, the presence of an intraparenchymal hematoma, the SEBES, the Hijdra score, and the GCS at arrival, was not significantly greater than the AUC of S100B alone $(p=0.946)$.

There were 12 patients with severe EBI and 69 controls (EBI-moderate + EBI-mild), allowing us to detect an AUC $>63 \%$ with a power of $95 \%$ and an alpha risk of $5 \%$. This value is lower than the lower limit of the $95 \%$ CI of the S100B AUC.

Among unconscious patients at admission (i.e., M-GCS $<6, n=25$ ), the effect size of the EBI-severe group on S100B was also large; Cliff's delta of 0.76 (95\%CI (0.34;0.93)). The AUC of S100B $(88.2 \% 95 \%$ 
CI $(72.2 ; 100))$ was significantly greater than that of the GCS at admission $(62.3 \%(53.1 ; 72.0), p=0.003)$ and of the Hijdra score $(53.8 \% 95 \% \mathrm{CI}(29.9 ; 77.8), p=0.012)$, and tended to be better than the AUC of the SEBES $(70.1 \% 95 \%$ CI $(50 ; 88.5), p=0.151)$. The AUC of a logistic regression model including S100B, the presence of an intraparenchymal hematoma, the SEBES, the Hijdra score, and the GCS at arrival was not significantly greater than the AUC of S100B alone $(p=0.77)$.

The best S100B thresholds and gray zone limits are described in Table 2 and highlighted on the ROC curves in Figure 2.

\subsection{Serum S100B Concentration and $m R S$ at ICU Discharge}

The median maximal serum S100B value at admission or day 1 was significantly different between the seven levels of the mRS $(p<0.001)$. Post hoc analyses found that median S100B values were significantly higher in patients with severe disability $(\mathrm{mRS}=5,0.302 \mu \mathrm{g} / \mathrm{L}, \mathrm{IQR}(0.216-0.531)$, $p=0.003$ ) or dead (mRS $=6,1.49 \mu \mathrm{g} / \mathrm{L}$, IQR $(0.957-3.1), p=0.003)$ compared to those without disability (i.e., $\mathrm{mRS}<2,0.093 \mu \mathrm{g} / \mathrm{L}(0.062-0.124))$. The maximal serum S100B value at admission or day $1 \mathrm{had}$ an AUC of $95.7 \%(95 \%$ CI $(90.52 ; 100))$ to predict an mRS > 4 (severe disability or death). A value $>0.262 \mu \mathrm{g} / \mathrm{L}(95 \% \mathrm{CI}(0.164 ; 0.494))$ predicted an mRS > 4 at ICU discharge with a $94.3 \%(95 \% \mathrm{CI}$ $(88.6 ; 98.61))$ specificity and $81.8 \%(95 \%$ CI $(54.6 ; 100))$ sensitivity. The gray zone of diagnosis ranged from $0.199 \mu \mathrm{g} / \mathrm{L}$ to $0.262 \mu \mathrm{g} / \mathrm{L}$. The maximal value of serum S100B was in this gray zone for $12.3 \%$ of patients $(n=10 / 81)$.

Among conscious patients at day 3 (EBI-mild and EBI-moderate groups, $n=69$ ), seven had an early S100B $>0.256 \mu \mathrm{g} / \mathrm{L}$ (upper gray zone threshold supposed to predict an M-GCS $<6$ with almost certainty), and were considered false positives. They were all Fisher 4 with a SEBES $>2$, and three had an intracerebral hematoma. They had a poor outcome (median (IQR) $\mathrm{mRS}=4(3-4.5)$ ) with frequent DCI occurrence $(n=5 / 7,71.4 \%)$.

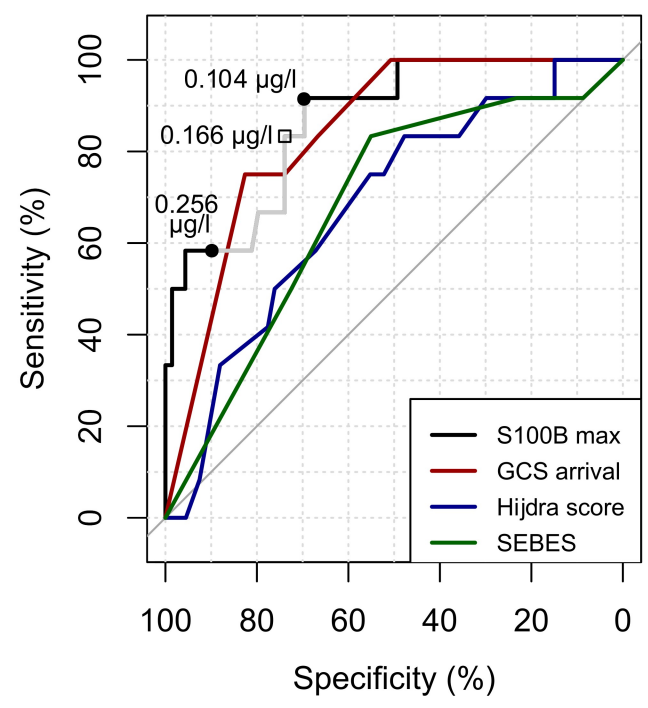

(a) All patients $(n=81)$

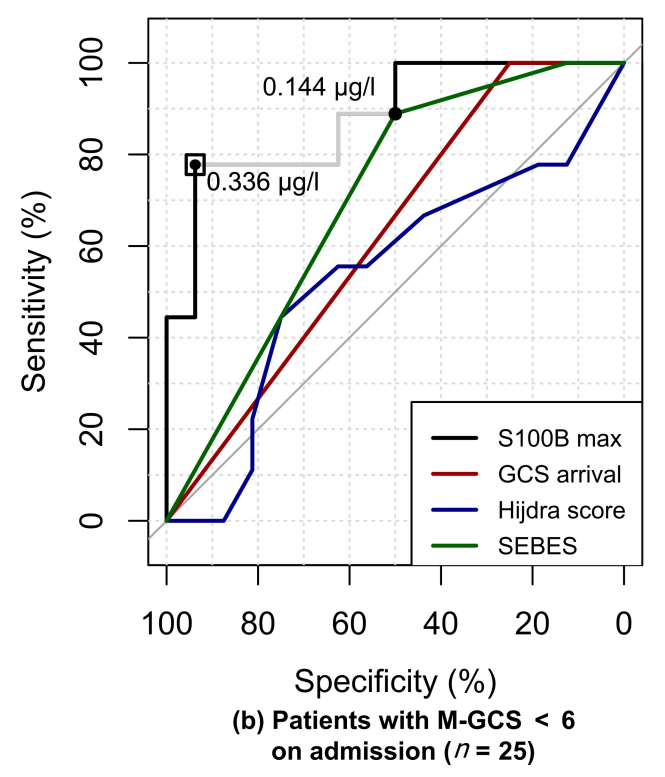

Figure 2. Receiver-operator characteristic (ROC) curves of early brain injury (EBI) biomarkers. (a) ROC analyses of the total population $(n=81)$. (b) ROC analyses of patients with a motor component of the Glasgow coma score $<6$ at admission $(n=25)$. Several S100B thresholds are plotted on both panels: (black square) the best threshold; (gray line) the gray zone of diagnostic uncertainty with (black dots) as its lower limit (i.e., to exclude severe EBI with almost certainty (Se $\geq 90 \%$ )) and upper limit (i.e., to predict severe EBI with almost certainty $(\mathrm{Sp} \geq 90 \%)$ ). 
Table 2. Accuracy of maximal S100B and GCS at ICU admission to predict an M-GCS $<6$ on day 3 in the whole cohort and only in patients with an M-GCS $<6$ at ICU admission.

\begin{tabular}{|c|c|c|c|c|}
\hline & \multicolumn{2}{|c|}{$\begin{array}{c}\text { All Patients } \\
(n=81, \text { M-GCS }<6 \text { at Day } 3 n=12)\end{array}$} & \multicolumn{2}{|c|}{$\begin{array}{l}\text { Patients with M-GCS }<6 \text { at Admission } \\
(n=25, \text { M-GCS }<6 \text { at Day } 3 n=9)\end{array}$} \\
\hline & S100B Max & GCS at Admission & S100B Max & GCS at Admission \\
\hline AUC, \% $(95 \%$ CI $)$ & $86.7(73.6 ; 95.9)$ & 84.1 (73.9-92.6) & $88.2(72.2 ; 100)$ & $62.5(53.1 ; 72.0)$ \\
\hline Best threshold $(95 \% \mathrm{CI})$ & $\begin{array}{c}0.165 \mu \mathrm{g} / \mathrm{L} \\
(0.100 ; 0.494)\end{array}$ & $4.5(4.5-14.5)$ & $\begin{array}{c}0.336 \mu \mathrm{g} / \mathrm{L} \\
(0.123 ; 0.951)\end{array}$ & $4.5(4.5 ; 8.5)$ \\
\hline Se, $\%(95 \%$ CI $)$ & $83.3(58.3 ; 100)$ & $75.0(50 ; 100)$ & $77.7(44.4 ; 100)$ & 100 \\
\hline Sp, \% (95\% CI) & $73.9(62.3 ; 84.1)$ & $82.6(73.9 ; 91.3)$ & $93.8(81.3 ; 100)$ & $25(6.3 ; 43.8)$ \\
\hline PLR $(95 \%$ CI) & $3.2(2.0 ; 5.1)$ & $4.3(2.3 ; 7.9)$ & $12.4(1.8 ; 85.7)$ & $1.3(0.9-1.8)$ \\
\hline NLR $(95 \%$ CI) & $0.23(0.06 ; 0.81)$ & $0.30(0.11 ; 0.81)$ & $0.24(0.07 ; 0.81)$ & $0.19(0.01 ; 3.14)$ \\
\hline Gray zone $\mu \mathrm{g} / \mathrm{L}$ & $0.104-0.256$ & $13.5-4.5$ & $0.144-0.336$ & $4.5-3$ \\
\hline Inter-LHR & $0.16-5.75$ & $0.25-4.32$ & $0.22-12.4$ & $1.2-0.19$ \\
\hline$\%$ in gray zone & 38 & 14 & 32 & 84 \\
\hline
\end{tabular}

The gray zones of diagnostic uncertainty are presented with the percentage of patients inside, and their interval likelihood ratio (inter-LHR). The positive LHR of the highest value of the biomarker in the gray zone is considered to include the diagnosis and the negative LHR of the lowest value to exclude the diagnosis. AUC: area under the curve, 95\% CI: 95\% confidence interval, Se: sensitivity, Sp: specificity, PLR: positive likelihood ratio, NLR: negative likelihood ratio.

\section{Discussion}

In this prospective blinded study, the maximal S100B serum concentration at admission and day 1 was found to have a good diagnostic value to predict severe consequences of EBI leading to an unconscious state at day 3 [31]. The early S100B had a better diagnostic accuracy than other EBI markers (i.e., clinical examination, SEBES, or Hijdra scores), in particular for patients unconscious at admission. To stratify the risk of severe EBI, we used a gray zone approach, defined by two thresholds: one to exclude (lower) and one to predict (upper) severe EBI with almost certainty. The lower threshold of the gray zone was close to the 95th percentile reported in healthy controls [33]. Values below this threshold are considered normal by the laboratory (Cobas, Roche, Mannheim, Germany). In the study population, given a $14.8 \%(n=12 / 81)$ pre-test probability of being unconscious at day 3 , an S100B level below the gray zone gives a $4 \%$ post-test probability, while an S100B level over this gray zone gives a $50 \%$ post-test probability (Figure 3 ). If patients were already unconscious at admission, the pre-test probability was $36 \%(n=9 / 25)$, thus an S100B level below the gray zone gives an $11 \%$ post-test probability, while an S100B level above the gray zone gives an $87 \%$ post-test probability. Using this approach, patients with an S100B level greater that the upper threshold, but with an M-GCS $=6$ at day 3, were considered as false positives. This S100B elevation could, in some patients, be explained by an intraparenchymal hematoma instead of severe EBI. Nevertheless, these patients had a high frequency of DCI and a poor neurological outcome at ICU discharge, suggesting a greater amount of brain injury, despite a reassuring early clinical presentation. Therefore, we propose that patient management strategy and EBI evaluation include an early S100B measurement, in addition to clinical examination and brain imaging. Patients with a low S100B level are expected to have mild EBI with good early and delayed outcomes, while those presenting with moderate or severe EBI are at risk of secondary injury and require close neurological monitoring. Taken together, these results suggest that physicians should adapt their neurological monitoring strategy in patients with moderate or severe EBI without delaying multimodal monitoring (e.g., continuous EEG, transcranial Doppler, or brain oxygen probe, Figure 3). 


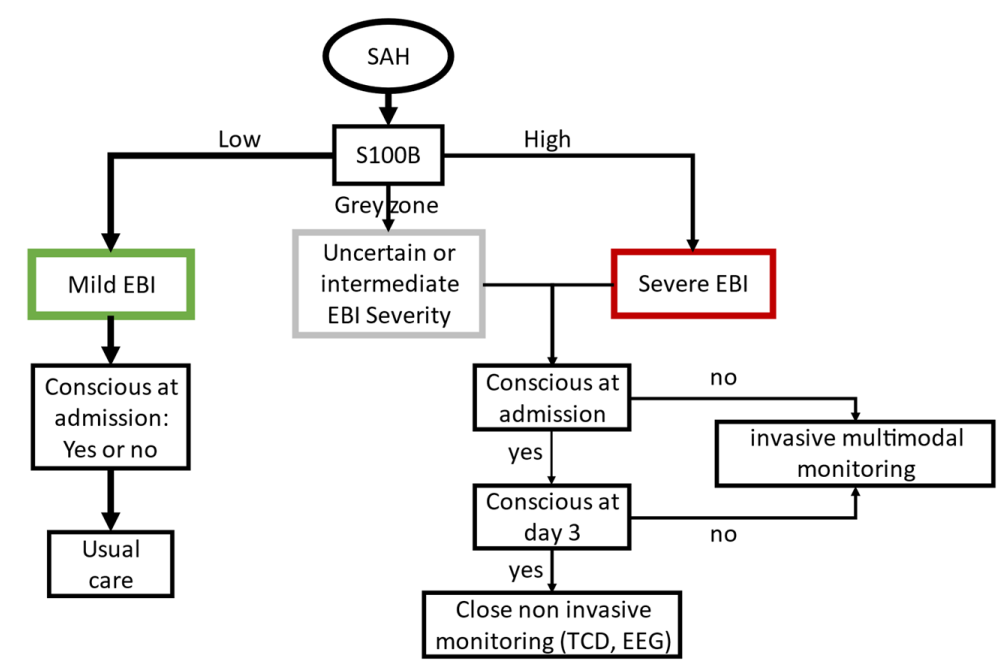

Figure 3. Algorithm chart for early brain injury severity prognostication. Early brain injury (EBI) severity prognostication and the proposed resulting management strategies are presented based on early S100B serum concentration. Low S100B: concentrations below the lower threshold of the gray zone rejecting severe EBI; high S100B: concentrations above the upper threshold of the gray zone predicting severe EBI. An S100B concentration within the gray zone has an uncertain diagnostic value but suggests significant brain injury. EEG: electroencephalography; ICH: intracerebral hematoma; TCD: transcranial doppler.

S100B serum concentration has previously been evaluated to predict long-term prognosis. Since the long-term outcome is the result of early and delayed injury, the peak or mean S100B values of the first week was found to have a good diagnostic value to predict poor long-term outcomes (AUC from $80 \%$ to $93 \%$ ) $[18,20,21]$. Unlike the values of the first week, early S100B has a poorer diagnostic accuracy to predict a poor outcome (i.e., $\mathrm{mRS}>2$ ) $[18,21,34]$. Herein, S100B was elevated only in patients with an $\mathrm{mRS}>4$ and was a good predictor of catastrophic outcome, such as death or disorder of consciousness. The early S100B values in patients with intermediate outcomes were more heterogeneous and this could explain why other authors found a poor diagnostic accuracy, as they aimed to predict mRS $>2$ (i.e., all levels of disability) $[18,21,34]$, whereas herein the cut-off was $\mathrm{mRS}>4$ (i.e., severe disability or death).

As evidenced by the sustained S100B elevation, patients with severe EBI undergo prolonged brain aggression leading to catastrophic outcomes. The pathophysiology of these brain injuries is yet to be elucidated, and S100B protein could by itself play a role in brain injury progression. The ratio between serum and cerebral spinal fluid S100B concentration is close to the one early after SAH [35]. Therefore, brain concentration is expected to reach the micromolar range, at which it can have neurotoxic effects [36]. S100B could thus be both a biomarker of brain damage and a damage-associated molecular pattern (DAMP) molecule. There is now growing evidence that DAMPs released upon the primary hemorrhage are involved in EBI and trigger cellular processes, such as neuro-inflammation, eventually leading to delayed ischemia [37]. DAMPs, such as the S100 family of proteins but also HMGB-1 or extracellular matrix-derived proteins, are released into the blood compartment and have been used as biomarkers of cerebral vasospasm, DCI, or long-term outcome [37-39]. Furthermore, HMGB-1 early concentration (i.e., at day 1 after the ictus) has been reported to predict the occurrence of cerebral vasospasm regardless of the initial severity [37]. Unlike HMGB-1, early S100B systemic concentration seems to not be predicative of DCI or cerebral vasospasm [21], and further evaluation of several biomarkers of EBI, including DAMPs and markers of cellular damage, are required to identify patient profiles.

Several limitations of this study must be noted. First, there were some missing measurements at admission or day 1. Nevertheless, even if S100B is known to have a short half-life both in the blood and brain extracellular space [40], we and others have found a sustained plasmatic elevation over the first 
$48 \mathrm{~h}$ following the ictus $[18,20]$; we therefore believe that a single measurement of S100B within the first day is representative of this period. Second, although the number of patients included was higher than that required according to power calculations, the small number of unconscious patients at admission limits the extrapolation of the results to all poor-grade SAH. This preliminary study will need an independent validation cohort to confirm the accuracy of the different thresholds that we suggest to be used at the bedside, in particular among patients with an M-GCS $<6$ at admission. Furthermore, we could not separately analyze patients with early complications, such as re-bleeding, in whom the S100B levels did not only reflect brain injury due to the primary hemorrhage. Third, the preference for coiling whenever possible in our institution may also limit the generalizability of the reported thresholds to institutions with a higher frequency of surgical procedures which are known to increase S100B levels [20]. With such a strategy, S100B values are expected to be higher even in those with mild or moderate EBI, and its diagnostic accuracy might thus be poorer with higher thresholds. Finally, since S100B is also expressed in melanocytes and Langerhans cells [41], its serum concentration is known to be higher in dark-skinned people, as well as after sun exposure [42,43]. Although we did not collect patient skin pigmentation, the population admitted to our institution is predominantly Caucasian.

\section{Conclusions}

This study provides evidence that S100B serum concentration within the first $24 \mathrm{~h}$ seems to have a good diagnostic value to predict severe EBI consequences, especially in patients unconscious at admission. Before claiming that $\mathrm{S} 100 \mathrm{~B}$ could thus be used as a surrogate for EBI severity to start earlier multimodal monitoring, these results must be confirmed in an independent validation cohort.

Author Contributions: Conceptualization, B.B., T.R., and F.D.; methodology, B.B., T.R., A.P.-L.; S100B data acquisition: C.R., L.D., A.P.-L., patient data acquisition T.R., F.G., C.B., R.C., A.T., F.D.; data analysis, B.B. and T.R.; writing-original draft preparation, B.B., T.R., F.G., A.P.-L., and F.D.; writing-review and editing, all authors; supervision, F.D. and A.P.-L.; project administration, F.D. All authors have read and agreed to the published version of the manuscript.

Funding: This research received no external funding.

Acknowledgments: The authors wish to acknowledge Philip Robinson, Department of Clinical Research and Innovation of the Hospices Civils de Lyon, for his help in writing the manuscript.

Conflicts of Interest: The authors declare no conflict of interest.

\section{References}

1. Ingall, T.; Asplund, K.; Mähönen, M.; Bonita, R. A Multinational Comparison of Subarachnoid Hemorrhage Epidemiology in the WHO MONICA Stroke Study. Stroke 2000, 31, 1054-1061. [CrossRef] [PubMed]

2. Connolly, S.E.; Rabinstein, A.A.; Carhuapoma, R.J.; Derdeyn, C.P.; Dion, J.; Higashida, R.T.; Hoh, B.L.; Kirkness, C.J.; Naidech, A.M.; Ogilvy, C.S.; et al. Guidelines for the Management of Aneurysmal Subarachnoid Hemorrhage A Guideline for Healthcare Professionals From the American Heart Association/American Stroke Association. Stroke 2012, 43, 1711-1737. [CrossRef] [PubMed]

3. Broderick, J.; Brott, T.; Duldner, J.; Tomsick, T.; Leach, A. Initial and recurrent bleeding are the major causes of death following subarachnoid hemorrhage. Stroke 1994, 25, 1342-1347. [CrossRef] [PubMed]

4. Claassen, J.; Vu, A.; Kreiter, K.T.; Kowalski, R.G.; Du, E.Y.; Ostapkovich, N.; Fitzsimmons, B.-F.M.; Connolly, E.; Mayer, S.A. Effect of acute physiologic derangements on outcome after subarachnoid hemorrhage. Crit. Care Med. 2004, 32, 832-838. [CrossRef] [PubMed]

5. Naidech, A.M.; Janjua, N.; Kreiter, K.T.; Ostapkovich, N.D.; Fitzsimmons, B.-F.F.; Parra, A.; Commichau, C.; Connolly, E.; Mayer, S.A. Predictors and impact of aneurysm rebleeding after subarachnoid hemorrhage. Arch. Neurol. 2005, 62, 410-416. [CrossRef]

6. Choi, H.; Bajgur, S.S.; Jones, W.H.; Savarraj, J.P.; Ko, S.-B.B.; Edwards, N.J.; Chang, T.R.; Hergenroeder, G.W.; Dannenbaum, M.J.; Chen, P.; et al. Quantification of Cerebral Edema After Subarachnoid Hemorrhage. Neurocritical Care 2016, 25, 64-70. [CrossRef]

7. Kreiter, K.T.; Copeland, D.; Bernardini, G.L.; Bates, J.E.; Peery, S.; Claassen, J.; Du, E.Y.; Stern, Y.; Connolly, S.E.; Mayer, S.A. Predictors of cognitive dysfunction after subarachnoid hemorrhage. Stroke 2002, 33. [CrossRef] 
8. Teasdale, G.; Drake, C.; Hunt, W.; Kassell, N.; Sano, K.; Pertuiset, B.; Villiers, D.J. A universal subarachnoid hemorrhage scale: Report of a committee of the World Federation of Neurosurgical Societies. J. Neurol. Neurosurg. Psychiatry 1988, 51, 1457. [CrossRef] [PubMed]

9. Suzuki, H. What is Early Brain Injury? Transl. Stroke Res. 2015, 6, 1-3. [CrossRef] [PubMed]

10. Rass, V.; Helbok, R. Early Brain Injury after Poor-Grade Subarachnoid Hemorrhage. Curr. Neurol. Neurosci. 2019, 19, 78. [CrossRef] [PubMed]

11. Francoeur, C.L.; Mayer, S.A. Management of delayed cerebral ischemia after subarachnoid hemorrhage. Crit. Care 2016, 20, 277. [CrossRef] [PubMed]

12. Helbok, R.; Madineni, R.C.; Schmidt, M.J.; Kurtz, P.; Fernandez, L.; Ko, S.-B.B.; Choi, A.; Stuart, M.R.; Connolly, E.; Lee, K.; et al. Intracerebral monitoring of silent infarcts after subarachnoid hemorrhage. Neurocritical Care 2011, 14, 162-167. [CrossRef] [PubMed]

13. Charpentier, C.; Audibert, G.; Guillemin, F.; Civit, T.; Ducrocq, X.; Bracard, S.; Hepner, H.; Picard, L.; Laxenaire, M. Multivariate analysis of predictors of cerebral vasospasm occurrence after aneurysmal subarachnoid hemorrhage. Stroke 1999, 30, 1402-1408. [CrossRef] [PubMed]

14. Ahn, S.-H.H.; Savarraj, J.P.; Pervez, M.; Jones, W.; Park, J.; Jeon, S.-B.B.; Kwon, S.U.; Chang, T.R.; Lee, K.; Kim, D.H.; et al. The Subarachnoid Hemorrhage Early Brain Edema Score Predicts Delayed Cerebral Ischemia and Clinical Outcomes. Neurosurgery 2017. [CrossRef] [PubMed]

15. Petzinka, V.N.; Endisch, C.; Streitberger, K.J.; Salih, F.; Ploner, C.J.; Storm, C.; Nee, J.; Leithner, C. Unresponsive wakefulness or coma after cardiac arrest-A long-term follow-up study. Resuscitation 2018. [CrossRef] [PubMed]

16. Calderon, L.M.; Guyette, F.X.; Doshi, A.A.; Callaway, C.W.; Rittenberger, J.C.; Service, P. Combining NSE and S100B with clinical examination findings to predict survival after resuscitation from cardiac arrest. Resuscitation 2014, 85, 1025-1029. [CrossRef] [PubMed]

17. Vos, P.; Gils, V.M.; Beems, T.; Zimmerman, C.; Verbeek, M. Increased GFAP and S100 $\beta$ but not NSE serum levels after subarachnoid haemorrhage are associated with clinical severity. Eur. J. Neurol. 2006, 13, 632-638. [CrossRef]

18. Quintard, H.; Leduc, S.; Ferrari, P.; Petit, I.; Ichai, C. Early and persistent high level of PS $100 \beta$ is associated with increased poor neurological outcome in patients with SAH: Is there a PS $100 \beta$ threshold for SAH prognosis? Crit. Care 2016, 20, 33. [CrossRef]

19. Foerch, C.; Niessner, M.; Back, T.; Bauerle, M.; Marchis, G.; Ferbert, A.; Grehl, H.; Hamann, G.F.; Jacobs, A.; Kastrup, A.; et al. Diagnostic Accuracy of Plasma Glial Fibrillary Acidic Protein for Differentiating Intracerebral Hemorrhage and Cerebral Ischemia in Patients with Symptoms of Acute Stroke. Clin. Chem. 2012, 58, 237-245. [CrossRef]

20. Weiss, N.; Sanchez-Peña, P.; Roche, S.; Beaudeux, J.L.; Colonne, C.; Coriat, P.; Puybasset, L. Prognosis value of plasma S100B protein levels after subarachnoid aneurysmal hemorrhage. Anesthesiology 2006, 104, 658-666. [CrossRef]

21. Lai, P.M.; Du, R. Association between S100B Levels and Long-Term Outcome after Aneurysmal Subarachnoid Hemorrhage: Systematic Review and Pooled Analysis. PLoS ONE 2016, 11, e0151853. [CrossRef] [PubMed]

22. Macdonald, R.L.; Higashida, R.T.; Keller, E.; Mayer, S.A.; Molyneux, A.; Raabe, A.; Vajkoczy, P.; Wanke, I.; Bach, D.; Frey, A.; et al. Randomized Trial of Clazosentan in Patients With Aneurysmal Subarachnoid Hemorrhage Undergoing Endovascular Coiling. Stroke 2012, 43, 1463-1469. [CrossRef] [PubMed]

23. Fisher, C.; Kistler, J.; Davis, J. Relation of cerebral vasospasm to subarachnoid hemorrhage visualized by computerized tomographic scanning. Neurosurgery 1980, 6, 1-9. [CrossRef] [PubMed]

24. Lindvall, P.; Runnerstam, M.; Birgander, R.; Koskinen, L.-O.D. The Fisher grading correlated to outcome in patients with subarachnoid haemorrhage. Br. J. Neurosurg. 2009, 23, 188-192. [CrossRef] [PubMed]

25. Hijdra, A.; Brouwers, P.J.; Vermeulen, M.; van Gijn, J. Grading the amount of blood on computed tomograms after subarachnoid hemorrhage. Stroke 1990, 21, 1156-1161. [CrossRef] [PubMed]

26. Molyneux, A.J.; Kerr, R.S.; Yu, L.-M.; Clarke, M.; Sneade, M.; Yarnold, J.A.; Sandercock, P.; Group, I. For the International subarachnoid aneurysm trial (ISAT) of neurosurgical clipping versus endovascular coiling in 2143 patients with ruptured intracranial aneurysms: A randomised comparison of effects on survival, dependency, seizures, rebleeding, subgroups, and aneurysm occlusion. Lancet 2005, 366, 809-817. [CrossRef] 
27. Bossuyt, P.M.; Reitsma, J.B.; Bruns, D.E.; Gatsonis, C.A.; Glasziou, P.P.; Irwig, L.; Lijmer, J.G.; Moher, D.; Rennie, D.; de Vet, H.C.; et al. STARD 2015: An updated list of essential items for reporting diagnostic accuracy studies. BMJ 2015, 351, h5527. [CrossRef]

28. Obuchowski, N.A.; McCLISH, D.K. Sample size determination for diagnostic accuracy studies involving binomial ROC curve indices. Stat. Med. 1997, 16, 1529-1542. [CrossRef]

29. Robin, X.; Turck, N.; Hainard, A.; Tiberti, N.; Lisacek, F.; Sanchez, J.-C.; Müller, M. pROC: An open-source package for R and $\mathrm{S}+$ to analyze and compare ROC curves. BMC Bioinform. 2011, 12, 1-8. [CrossRef]

30. Cliff, N. Ordinal Methods for Behavioral Data Analysis, 1st ed.; Psychology Press: New York, NY, USA, 1996. [CrossRef]

31. Ray, P.; Manach, Y.; Riou, B.; Houle, T.T. Statistical Evaluation of a Biomarker. Anesthesiology 2010, $112,1023$. [CrossRef] [PubMed]

32. R Core Team. R: A Language and Environment for Statistical Computing; R Foundation for Statistical Computing: Vienna, Austria, 2017.

33. Biberthaler, P.; Linsenmeier, U.; Pfeifer, K.-J.; Kroetz, M.; Mussack, T.; Kanz, K.-G.; Hoecherl, E.F.J.; Jonas, F.; Marzi, I.; Leucht, P.; et al. Serum S-100B concentration provides additional information for the indication of computed tomography in patients after minor head injury. Shock 2006, 25, 446-453. [CrossRef] [PubMed]

34. Sanchez-Peña, P.; Pereira, A.-R.; Sourour, N.-A.; Biondi, A.; Lejean, L.; Colonne, C.; Boch, A.-L.; Hawari, M.; Abdennour, L.; Puybasset, L. S100B as an additional prognostic marker in subarachnoid aneurysmal hemorrhage. Crit. Care Med. 2008, 36, 2267-2273. [CrossRef] [PubMed]

35. Kleindienst, A.; Meissner, S.; Eyupoglu, I.Y.; Parsch, H.; Schmidt, C.; Buchfelder, M. Dynamics of S100B Release into Serum and Cerebrospinal Fluid Following Acute Brain Injury. In Brain Edema XIV; Springer: Vienna, Austria, 2009; pp. 247-250. [CrossRef]

36. Bianchi, R.; Kastrisianaki, E.; Giambanco, I.; Donato, R. S100B Protein Stimulates Microglia Migration via RAGE-dependent Up-regulation of Chemokine Expression and Release. J. Biol. Chem. 2011, 286, 7214-7226. [CrossRef] [PubMed]

37. Chaudhry, S.R.; Güresir, A.; Stoffel-Wagner, B.; Fimmers, R.; Kinfe, T.M.; Dietrich, D.; Lamprecht, A.; Vatter, H.; Güresir, E.; Muhammad, S. Systemic High-Mobility Group Box-1. Crit. Care Med. 2018, 46, e1023-e1028. [CrossRef] [PubMed]

38. Chaudhry, S.; Hafez, A.; Jahromi, B.; Kinfe, T.; Lamprecht, A.; Niemelä, M.; Muhammad, S. Role of Damage Associated Molecular Pattern Molecules (DAMPs) in Aneurysmal Subarachnoid Hemorrhage (aSAH). Int. J. Mol. Sci. 2018, 19, 2035. [CrossRef] [PubMed]

39. Bell, J.D.; Rhind, S.G.; Battista, A.P.D.; Macdonald, R.L.; Baker, A.J. Biomarkers of Glycocalyx Injury are Associated with Delayed Cerebral Ischemia Following Aneurysmal Subarachnoid Hemorrhage: A Case Series Supporting a New Hypothesis. Neurocrit. Care 2017, 26, 339-347. [CrossRef] [PubMed]

40. Sen, J.; Belli, A.; Petzold, A.; Russo, S.; Keir, G.; Thompson, E.; Smith, M.; Kitchen, N. Extracellular fluid S100B in the injured brain: A future surrogate marker of acute brain injury? Acta Neurochir. 2005, 147, 897-900. [CrossRef]

41. Böni, R.; Burg, G.; Doguoglu, A.; Ilg, E.C.; Schäfer, B.W.; Müller, B.; Heizmann, C.W. Immunohistochemical localization of the Ca2+ binding S100 proteins in normal human skin and melanocytic lesions. Br. J. Derm. 1997, 137, 39-43. [CrossRef]

42. Bouvier, D.; Duret, T.; Rouzaire, P.; Jabaudon, M.; Rouzaire, M.; Nourrisson, C.; Bourgne, C.; Pereira, B.; Evrard, B.; Sapin, V. Preanalytical, analytical, gestational and pediatric aspects of the S100B immuno-assays. Clin. Chem. Lab. Med. 2016, 54, 833-842. [CrossRef]

43. Dadas, A.; Washington, J.; Marchi, N.; Janigro, D. Improving the clinical management of traumatic brain injury through the pharmacokinetic modeling of peripheral blood biomarkers. Fluids Barriers CNS 2016, 13, 21. [CrossRef]

(C) 2020 by the authors. Licensee MDPI, Basel, Switzerland. This article is an open access article distributed under the terms and conditions of the Creative Commons Attribution (CC BY) license (http://creativecommons.org/licenses/by/4.0/). 